pressure management following recanalization may play a role in improving clinical outcomes for these patients. Hence, we believe that there is a need for future prospective trials addressing this issue.

Disclosures S. Mannava: None. A. Garg: None.

\section{E-015 EXTRA-FEMORAL ACCESS FOR MECHANICAL THROMBECTOMY IN ACUTE ISCHEMIC STROKE}

${ }^{1} \mathrm{~N}$ Haranhalli, ${ }^{1} \mathrm{D}$ Altschul, ${ }^{2} \mathrm{D}$ Pasquale. ${ }^{1}$ Department of Neurological Surgery, Montefiore Medical Center, Bronx, NY; ${ }^{2}$ Department of Radiology, Montefiore Medical Center, Bronx, NY

\subsection{6/neurintsurg-2016-012589.87}

Objective To demonstrate the safety and effectiveness of extrafemoral endovascular access for mechanical thrombectomy for acute ischemic stroke (AIS) in patients whose vascular anatomy precludes safe or maneuverable trans-femoral access.

Methods We present a case series of seven patients treated by four separate neurointerventionalists utilizing either trans-radial or trans-cervical carotid access for treatment of acute ischemic stroke. All cases, except for one, were performed at Montefiore Medical Center, Bronx NY.

Results All seven patients presented with AIS symptoms and initial NCCT revealed no contraindications to proceeding for mechanical thrombectomy. Patients ranged from 25 to 88 years of age. There were two cases of basilar artery occlusion, two right middle cerebral artery (MCA) occlusions, two left MCA occlusions and one patient with a left carotid terminus occlusion. Femoral access was attempted in all but two patients, and sheath placement was successful in these five cases. In two of these five cases however, femoral catheterization was aborted after sheath placement due to identification of impassable femoral or aortic vascular anatomy; a prior femfem bypass in one and bilateral common femoral artery occlusions in another. The most common reason for aborting femoral access for thrombectomy was vessel tortuosity impeding catheterization of intracranial segments of either anterior or posterior circulation vessels. Four patients were treated via radial artery access and three patients were treated via cervical carotid access. Recanalization was achieved in six out of the seven patients. In five of the patients a TICI $2 \mathrm{~B} / 3$ recanalization score was achieved, and in one patient a TICI 2 A. There were no immediate procedure related complications observed. Two patients progressed to hemorrhagic conversion of their prior infarcts. No new acute infarcts were noted in any patient. Two patients expired several days post-thrombectomy due to cardiopulmonary arrest unrelated to intervention. Conclusions While trans-femoral access remains the mainstay for endovascular treatment of AIS, risk of vascular injury and delay of recanalization should alert the interventionalist to consider extra-femoral approaches. This case series demonstrates the safety and success possible with trans-radial or trans-cervical carotid catheterization in the setting of mechanical thrombectomy for AIS. With these findings, we feel strongly that further standardization of these techniques, guidelines for the need of extra-femoral access prospectively, and development of devices tailored for trans-radial and transcervical carotid approaches are indispensable to see significant advancements in the field of interventional stroke treatment.

Disclosures N. Haranhalli: None. D. Altschul: None. D. Pasquale: None.

\section{E-016 AN ATHEROSCLEROTIC PLAQUE PHANTOM FOR MEDICAL IMAGING}

${ }^{1} \mathrm{~J}$ Chueh, ${ }^{2} \mathrm{~T}$ Turan, ${ }^{1} \mathrm{~K}$ van der Marel, ${ }^{2} \mathrm{~T}$ LeMatty, ${ }^{3} \mathrm{~T}$ Brown, ${ }^{4} \mathrm{~S}$ Ansari, ${ }^{5} \mathrm{~T}$ Carroll, ${ }^{6} \mathrm{~A}$ Buck, ${ }^{7} X$ Zhou, ${ }^{3} \mathrm{~A}$ Chatterjee, ${ }^{1} \mathrm{R}$ King, ${ }^{1} \mathrm{~S}$ Zheng, ${ }^{8} \mathrm{R}$ Swartz, ${ }^{9} \mathrm{E}$ Feldmann, ${ }^{1} \mathrm{M}$ Gounis. ${ }^{1}$ Department of Radiology, UMass Medical School, Worcester, MA; ${ }^{2}$ Department of Neurology, Medical University of South Carolina Stroke Division, Charleston, SC; ${ }^{3}$ Department of Radiology and Radiological Science, Medical University of South Carolina, Charleston, SC; ${ }^{4}$ Department of Radiology, Neurology, and Neurosurgery, Northwestern University Feinberg School of Medicine, Chicago, IL; ${ }^{5}$ Department of Radiology, The University of Chicago, Chicago, IL; ${ }^{6}$ Department of Radiology and Radiological Sciences, Vanderbilt University, Nashville, $T N_{;}{ }^{7}$ Department of Radiology, Neurosurgery, and Bioengineering, University of Illinois College of Medicine, Chicago, IL; ${ }^{8}$ Sunnybrook Health Sciences Center, Toronto, ON, Canada; ${ }^{9}$ Department of Neurology, Baystate Medical Center, Springfield, MA

\subsection{6/neurintsurg-2016-012589.88}

Introduction Intracranial atherosclerotic disease (ICAD) is a common cause of ischemic stroke, but little is known about how the characteristics of intracranial plaques are related to stroke risk. Recently, studies have attempted to correlate ICAD lesions with high-resolution MRI (HR MRI) vessel wall findings, an important emerging technology, to identify various plaque components. However, long-term, multi-center clinical studies are needed to show if HR MRI plaque components contribute to stroke risk. The goal of this study is to build an ICAD phantom that incorporates materials mimicking a stenotic vessel and plaque components (fibrous cap and lipid core) for standardizing MRI pulse sequences across multiple imaging platforms necessary for development of multi-center ICAD HR MRI networks.

Materials and methods HRMRI data from a patient with a basilar artery plaque was used to acquire the detailed structure of the stenotic artery and plaque components. A virtual coreshell mold of the basilar artery plaque was $3 \mathrm{D}$ printed to form a physical object. During 3D printing, the volume and shape of each plaque component were defined in the model. Polyvinyl alcohol hydrogel was infused into the core-shell mold to form the stenotic artery. A fibrous cap was constructed using a mixture of agarose, carrageenan, sodium azide, and water. The lipid core was mimicked using vegetable fat, sodium azide, and carrageenan mixture. Two phantoms were manufactured and scanned using various $3 \mathrm{~T}$ MRI systems across 7 different sites for image quality assessment. Quantitative comparisons of the scan results for both structural dimensions of plaque components (e.g. lumen diameter) and contrast-to-noise ratio (CNR) were based on the thin cross-sectional slices from 3D T2-weighted TSE/FSE sequences.

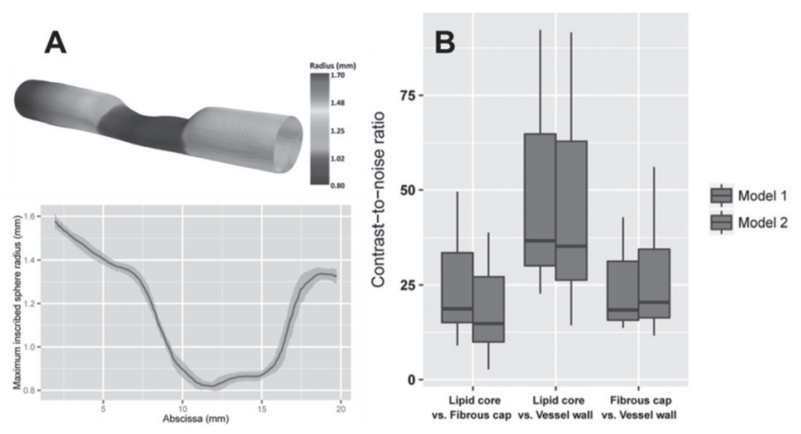

Abstract E-016 Figure 1 
Results HRMRI provides a detailed benchmark for validation of lumen geometry (Figure 1A, top). Quantitative evaluation of the lumen diameter in the vicinity of the stenosis using imaging data acquired from 7 different centers is presented as weighted mean and standard deviation (Figure 1A, bottom). It was observed that along the centerline of the lumen, the radius ranged from $0.8-1.6 \mathrm{~mm}$ and was highly reproducible across all imaging platforms. The highest CNRs were observed for comparisons between lipid and vessel wall (Figure 1B). CNR mean variation between the two phantom models for each comparison between plaque components was 3.68-6.09, demonstrating excellent reliability in manufacturing technique.

Conclusion A plaque phantom composed of a stenotic vessel wall and plaque components, including fibrous cap and a lipid core, was successfully constructed for multi-center HRMRI standardization.

Disclosures J. Chueh: None. T. Turan: None. K. van der Marel: None. T. LeMatty: None. T. Brown: None. S. Ansari: None. T. Carroll: None. A. Buck: None. X. Zhou: None. A. Chatterjee: None. R. King: None. S. Zheng: None. R. Swartz: None. E. Feldmann: None. M. Gounis: None.

\section{E-017 PURE TENTORIAL SUBDURAL HEMATOMA FROM RUPTURE OF ANEURYSM ALONG THE TRANSMASTOID BRANCHES OF THE OCCIPITAL ARTERY}

H Nguyen, N Doan, S Shabani, M Gelsomino, O Zaidat. Neurosurgery, Medical College of Wisconsin, Milwaukee, WI

\subsection{6/neurintsurg-2016-012589.89}

Background Pure subdural hematoma (without subarachnoid, intraventricular, or intraparenchymal hemorrhage) due to a rupture intracranial aneurysm is a rare pathology. Most reported cases involve an aneurysm along the internal carotid artery, posterior communicating artery, or middle cerebral artery. No reports have documented an aneurysm along the intracranial portion of a branch from the occipital artery.

Case presentation Patient is a 70 year old female, history of hypertension, congestive heart failure, renal artery stenosis, gout, who presented with sudden onset severe excruciating headaches, associated with spinning sensation, nausea, and emesis. No history of trauma. Neurological examination was unremarkable. CT head demonstrated a tentorial subdural hematoma. CTA head revealed a $4 \mathrm{~mm}$ aneurysm along the peripheral left inferior cerebelllum and $3 \mathrm{~mm}$ aneurysm adjacent to the right sigmoid sinus, both without clear visualization of the parent vessels. Diagnostic angiogram revealed

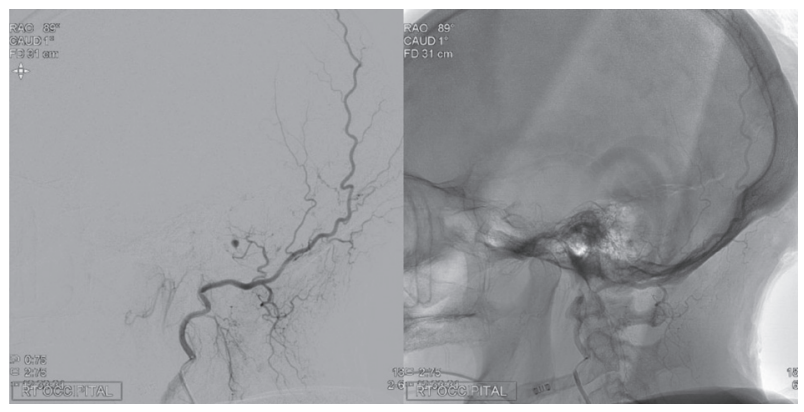

bilateral aneurysms along the transmastoid branches of the intracranial portion of both occipital arteries. Consequently, these branches were embolized, with no residual filling of the aneurysms. Post-procedure, she remained neurologically well. She was monitored appropriately for vasospasm, and discharged home 10 days after presentation.

Conclusion Rupture of aneurysms along intracranial branches of the occipital artery can lead to pure subdural hematoma along the tentorium. For non-traumatic acute subdural hematoma, vigilance should be directed towards a vascular etiology. Disclosures H. Nguyen: None. N. Doan: None. S. Shabani: None. M. Gelsomino: None. O. Zaidat: None.

\section{E-018 TREATMENT OF RUPTURED BLOOD-BLISTER-LIKE ANEURYSMS IN THE SUBACUTE PHASE: CLINICAL AND ANGIOGRAPHIC OUTCOME}

F Di Maria, J Gabrieli, B Bartolini, S Pistocchi, J Chiras, N Sourour, F Clarençon. Neuroradiology, GH Pitié Salpêtrière, Paris, France

\subsection{6/neurintsurg-2016-012589.90}

Purpose Ruptured Blood blisterlike (BBL) aneurysms represent a therapeutic challenge. Timing of treatment and technique of choice are still a subject of debate. We report our experience in the endovascular treatment of such lesions in the subacute phase.

Methods Between June 2011 and January 2015, 6 ruptured BBL aneurysms were treated at our institution. Four were located in the carotid siphon, 2 in the posterior circulation. Endovascular procedures were carried out between day 7 and day 15 after the hemorrhagic event. One patient was treated surgically. Flow-diverter stents (FDS) were used in 4 cases. Two telescopic laser-cut stents were used in one case. Double antiplatelet therapy was started 4 days before treatment in 1 case and the day of the procedure in the remaining 4 cases. Angiographic follow-up was carried out by MRA and DSA at 1 month, 6 months and 1 year.

Results All endovascular procedures were performed without technical difficulties. Antiplatelet treatment was started 4 days prior to procedure in one case and the day of the intervention in the other cases. One patient presented a transient motor deficit at day 1 after treatment. One patient had a peroperative cerebellar ischemia after FDS deployment, despite antiplatelet treatment. One patient treated by surgery had a fatal brain ischemia after peroperative aneurysmal rupture that eventually required clipping of the carotid siphon. Imaging follow was available for the remaining 5 patients. No patent rebled prior to treatment or during follow-up. Three out of five aneurysms were completely occluded at latest follow-up.

Conclusion Modern endovascular techniques for the treatment of ruptures BBL aneurysms, including the use of flow-diversion, seem promising. Treatment in the subacute phase may be considered as an option in relation to other clinical issues (patient WFNS grade, risk of rebleed under antiplatelet therapy, ventricular shunting) when pondering overall risks and benefits in patient management.

Disclosures F. Di Maria: None. J. Gabrieli: None. B. Bartolini: None. S. Pistocchi: None. J. Chiras: None. N. Sourour: None. F. Clarençon: None. 